\title{
Differential expression of miRNAs in the nervous system of a rat model of bilateral sciatic nerve chronic constriction injury
}

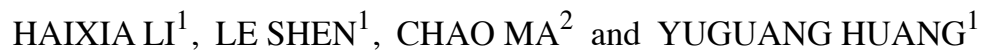 \\ ${ }^{1}$ Department of Anesthesiology, Peking Union Medical College Hospital; ${ }^{2}$ Department of Anatomy, \\ Histology and Embryology, Institute of Basic Medical Sciences, Chinese Academy of Medical Sciences, \\ School of Basic Medicine, Peking Union Medical College, Beijing 100730, P.R. China
}

Received February 17, 2013; Accepted April 17, 2013

DOI: $10.3892 /$ ijmm.2013.1381

\begin{abstract}
Chronic neuropathic pain is associated with global changes in gene expression in different areas of the nociceptive pathway. MicroRNAs (miRNAs) are small ( 22 nt long) noncoding RNAs, which are able to regulate hundreds of different genes post-transcriptionally. The aim of this study was to determine the miRNA expression patterns in the different regions of the pain transmission pathway using a rat model of human neuropathic pain induced by bilateral sciatic nerve chronic constriction injury (bCCI). Using microarray analysis and quantitative reverse transcriptase-PCR, we observed a significant upregulation in miR-341 expression in the dorsal root ganglion (DRG), but not in the spinal dorsal horn (SDH), hippocampus or anterior cingulate cortex (ACC), in the rats with neuropathic pain compared to rats in the naïve and sham-operated groups. By contrast, the expression of miR-203, miR-181a-1* and miR-541* was significantly reduced in the SDH of rats with neuropathic pain. Our data indicate that miR-341 is upregulated in the DRG, whereas miR-203, miR-181a-1* and miR-541* are downregulated in the SDH under neuropathic pain conditions. Thus, the differential expression of miRNAs in the nervous system may play a role in the development of chronic pain. These observations may aid in the development of novel treatment methods for neuropathic pain, which may involve miRNA gene therapy in local regions.
\end{abstract}

\section{Introduction}

Neuropathic pain is defined as pain arising as a direct consequence of a lesion or disease affecting either the peripheral or

Correspondence to: Professor Yuguang Huang, Department of Anesthesiology, Peking Union Medical College Hospital, No 1 Shuaifuyuan Wangfujing, Dongcheng, Beijing 100730, P.R. China E-mail: pumchhyg@yahoo.com.cn

Abbreviations: bCCI, bilateral sciatic nerve chronic constriction injury; SDH, spinal dorsal horn; DRG, dorsal root ganglion; ACC, anterior cingulate cortex

Key words: neuropathic pain, miRNA array, real-time PCR, nociceptive pathway central nervous system $(1,2)$. Although pain has been investigated in depth for decades, neuropathic pain is still frequently under-treated (3), due to poor understanding of its pathophysiological and molecular mechanisms. Several regions of the nociceptive pathway, including the anterior cingulate cortex (ACC), hippocampus, spinal dorsal horn (SDH) and dorsal root ganglion (DRG), are involved in the development and maintenance of neuropathic pain (4-9). Several recent studies have shown that peripheral and central sensitization are associated with global changes in gene expression in different regions of the pain transmission pathway, and that these changes may be part of the mechanisms behind neuropathic pain (10-13). In order to elucidate the molecular mechanisms underlying neuropathic pain, it is essential to determine how gene expression patterns are altered by nerve injuries and how these alterations lead to the development and maintenance of chronic pain.

miRNAs are a large class of short non-coding RNAs ( $\sim 22$ nt long), many of which are expressed either predominantly or exclusively in the nervous system (14-21). Furthermore, changes (either increases or decreases) in miRNA expression have been found in many disease states (22-24). Bilateral sciatic nerve chronic constriction injury (bCCI) is commonly used as a model for studying human neuropathic pain, in which chromic gut sutures are used to ligate each sciatic nerve. This model is characterized by long-lasting cold allodynia and mechanical hypersensitivity $(25,26)$; however, no information is available on how miRNA expression patterns in the nociceptive system are altered.

The aim of the present study was to examine the differential expression patterns of miRNAs in the DRG, SDH, hippocampus and ACC using a rat model of neuropathic pain induced by bCCI. Utilizing a microarray platform, we identified miRNAs with a 2-fold change in expression in the DRG and SDH due to bCCI. We then confirmed the results in the DRG, SDH, hippocampus and ACC using quantitative reverse transcriptase-polymerase chain reaction (qRT-PCR). Our results demonstrate that miRNAs are differentially expressed at the neuroanatomical level under neuropathic pain conditions in vivo.

\section{Materials and methods}

Animals. Specific pathogen-free (SPF) adult female Sprague-Dawley rats (Shanghai Laboratory, Animal Research 
Center, Shanghai, China) weighing 150-180 g were randomly divided into 3 groups (naïve, sham-operated and bCCI) and housed 2-3 per cage in a climate-controlled environment on a $12 \mathrm{~h}$ light/dark cycle with food and water ad libitum. Behavioral experiments were performed between 9:00 a.m. and 4:00 p.m. Animal handling and experimental procedures were performed in accordance with the policies and recommendations of the Guide for the Care and Use of Laboratory Animals, and were approved by the Ethics Committee for Animal Experimentation of the Peking Union Medical College Hospital, Beijing, China. The minimum number of rats was used and every effort was made to reduce their discomfort and stress.

Neuropathic pain model. bCCI was induced under aseptic conditions in rats anesthetized using a mixture of ketamine and xylazine (60 and $8 \mathrm{mg} / \mathrm{kg}$, respectively). The sciatic nerve on each side was exposed via a mid-thigh incision followed by the separation of the heads of the biceps femoris muscle. Each sciatic nerve was identified above the trifurcation and freed from the surrounding loose connective tissue before 4 snug ligatures of 4-0 chromic gut suture were placed around them. The sham-operated animals had their sciatic nerves exposed but not ligated, and the rats in the naïve group were not operated upon.

Mechanical withdrawal test. The thresholds for paw withdrawal in response to mechanical stimuli were assessed using Von Frey filaments at 1,2 and 3 days pre-operation and 1,3,7 and 14 days post-operation according to previously described procedures (27). At the end of the behavioral testing, rats were euthanized and the bilateral L4-6 DRG, L2-4 SDH, hippocampus and ACC were chronologically harvested and rapidly frozen at $-180^{\circ} \mathrm{C}$.

Acetone test. Cold allodynia was evaluated on 3 continuous days pre-operation and on days 1, 3, 7 and 14 post-operation. A drop $(0.1 \mathrm{ml})$ of room temperature acetone was gently applied to each hindpaw through polyethylene (PE; (10 standard specification) plastic tubing connected to a 1-ml syringe. A rapid withdrawal of the hindpaw in response to the spread of the acetone over the plantar surface was considered a sign of cold allodynia. The test was repeated 5 times for each hindpaw (alternating hindpaws) for a total of 10 trials/day with an interval of $\sim 2$ min between each test. The results were graded as a percentage of applications that evoked a response of hindpaw withdrawal. An increase in the percentage of applications that elicited a withdrawal response compared to the control was interpreted as the development of increased cold sensitivity.

RNA isolation. Total RNA was isolated using TRIzol reagent (Invitrogen Life Technologies, Carlsbad, CA, USA) and a miRNeasy mini kit (Qiagen, Hilden, Germany) in accordance with the manufacturer's instructions. This procedure efficiently recovered all RNA species, including miRNAs. RNA quality and quantity were measured using a NanoDrop spectrophotometer (ND-1000; NanoDrop Technologies, Wilmington, DE, USA), and RNA integrity was evaluated by gel electrophoresis.

Microarray analysis. Following RNA isolation, the miRCURY ${ }^{\mathrm{TM}} \mathrm{Hy}^{\mathrm{TM}} / \mathrm{Hy} 5^{\mathrm{TM}}$ Power labeling kit (Exiqon,
Vedbaek, Denmark) was used according to the manufacturer's specifications for miRNA labeling. Once the labeling procedure was completed, the Hy3-labeled samples were hybridized on a miRCURY LNA Array (v.16.0) (Exiqon) according to the manufacturer's instructions. Scanned images of the hybridized arrays were then imported into GenePix Pro 6.0 software (Axon) for grid alignment and data extraction. Replicated miRNAs were averaged, and miRNAs with intensities $\geq 50$ in all samples were chosen for calculating the normalization factor. Expression results were normalized using a median normalization. After normalization, significantly differentially expressed miRNAs (as determined by ANOVA followed by a Student-Newman-Keuls multiple comparison test) were identified through volcano plot filtering.

qRT-PCR analysis of miRNAs. Two-fold increases or decreases in gene expression observed in the microarrays were validated by qRT-PCR analysis. Complementary DNA (cDNA) was generated from $20 \mathrm{ng}$ of total RNA using a universal cDNA synthesis kit (Exiqon). The cDNA template was then amplified using mature miRNA-specific $\mathrm{LNA}^{\mathrm{TM}}$-enhanced forward and reverse primers (rno-miR-341\#130384, rno-miR203\#204285, rno-miR-181a-1*\#204110 and rno-miR-541\#130392; rno-RNU5G\#203908, Exiqon). A SYBR ${ }^{\circledR}$-Green Master mix kit (Exiqon) was used for detection. Real-time PCR was performed on an ABI PRISM ${ }^{\circledast} 7500$ Sequence Detection System (Applied Biosystems, Foster City, CA, USA) and data were analyzed using ABI PRISM 7500 Sequence Detection System Software, version 2.0.1 (Applied Biosystems). Levels of mature miRNAs (miR-341, miR-203, miR-181a-1* and miR-541*) in the DRG, $\mathrm{SDH}$, hippocampus and ACC were calculated in relation to the levels of U5 RNA (used as an internal control) using the $2^{-\Delta \Delta \mathrm{Ct}}$ method as previously described (28). Samples from the naïve group were used as a calibrator. Each set of PCR reactions included a no-template control and an RNA spike-in (a synthetic control template). We analyzed 5 biological replicates and 3 technical replicates. The ratios of miRNA amounts were compared among the samples using a one-way analysis of variance (ANOVA) followed by a Student-Newman-Keuls multiple comparison test.

Statistical analysis. The data for the mechanical threshold are expressed as the means \pm SEM. Kruskal-Wallis ANOVA was used to analyze the differences among treatment groups. Acetone test data were analyzed using a Pearson's $\chi^{2}$ test. The changes in miRNA expression among the groups were analyzed by ANOVA followed by a Student-Newman-Keuls multiple comparison test. All statistical tests were performed using SPSS version 17.0 software. Data were analyzed and are expressed as the means $\pm \mathrm{SEM}$, as stated in the figures.

\section{Results}

Mechanical hypersensitivity test. The mechanical sensitivity threshold of the rats in the bCCI group on post-operative days 7 and 14 was significantly lower than that in the naïve and sham-operated group rats (Fig. 1; Kruskal-Wallis ANOVA, $\mathrm{P} \leq 0.001)$. The responses were not significantly different among the groups on pre-operative days or postoperative days 1 or 3 . 
A

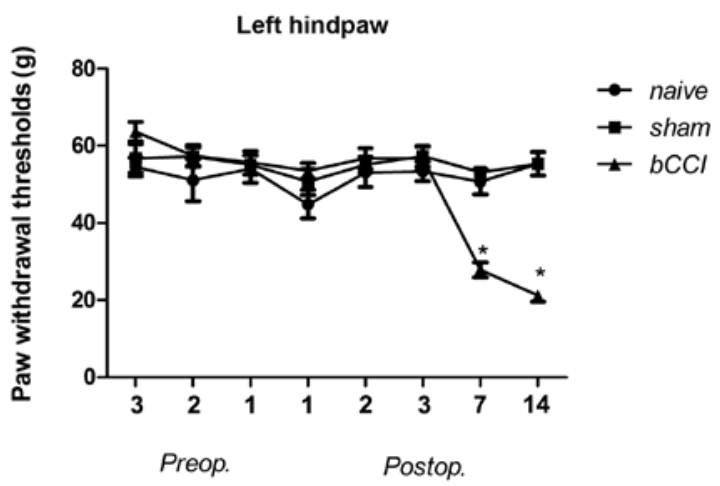

Time (day)

B

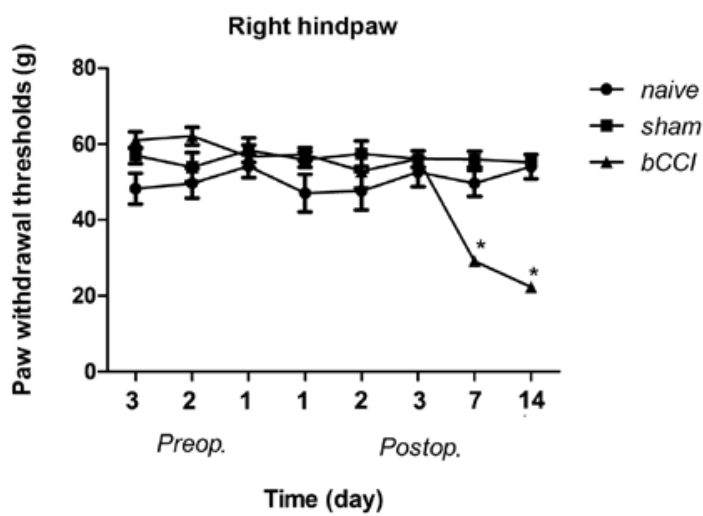

Figure 1. Paw withdrawal thresholds for naïve, sham-operated and bilatera sciatic nerve chronic constriction injury (bCCI) group rats used in microarray analysis and qRT-PCR. (A) Left hindpaw, (B) right hindpaw. Rats submitted to sciatic ligation developed tactile stimulus-induced hypersensitivity at 7 and 14 days post-surgery, whereas the naïve and sham-operated group rats had no change in their sensitivity ("Kruskal-Wallis ANOVA, $\mathrm{P} \leq 0.001$ ). Preop, pre-operation; Postop, post-operation.

Acetone test. The bCCI group rats displayed cold allodynia on days 7 and 14 post-ligation, whereas the sham-operated and naïve group rats showed no change as regards cold allodynia (Fig. 2; Pearson's $\chi^{2}$ test $\mathrm{P}<0.001$ ).

Microarray analysis. Using miRCURY LNA expression arrays we found that the expression of miR-341 was substantially upregulated in the DRG of the bCCI group rats compared to the sham-operated and naïve group rats (Table I; one-way ANOVA, fold change $>2, \mathrm{P}<0.05)$. By contrast, the expression of miR-203, miR-181a-1* and miR-541* in the SDH of the bCCI group rats was significantly downregulated compared to the sham-operated and naïve group rats (Table II; one-way ANOVA, fold change $>2, \mathrm{P}<0.05$ ).

$q R T-P C R$ validation of the differentially expressed miRNAs in the pain transmission pathway. Using RT-PCR, we analyzed miR-341, miR-203, miR-181a-1* and miR-541* expression in total RNA isolated from the DRG, $\mathrm{SDH}$, hippocampus and ACC from the naïve, sham-operated and bCCI group rats ( $\mathrm{n}=5$ for each category). A comparison of the relative miRNA expression levels among the different treatment groups revealed a significant upregulation of miR-341 in the DRG of the bCCI group rats, in agreement with the microarray data.
$\mathbf{A}$

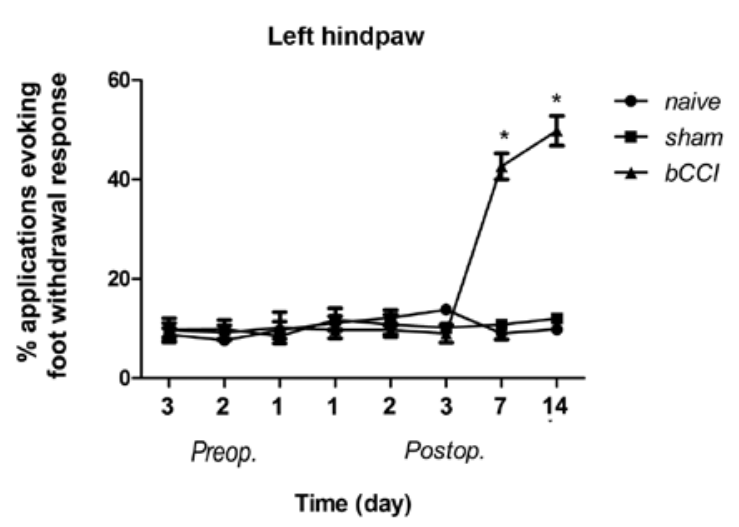

B

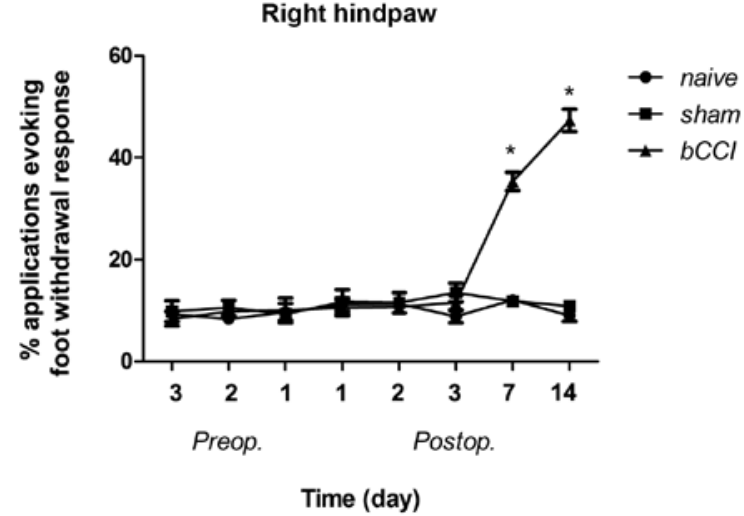

Figure 2. The incidence of cold allodynia for the naïve, sham-operated and bilateral sciatic nerve chronic constriction injury (bCCI) group rats used in microarray analysis and qRT-PCR. (A) Left hindpaw, (B) right hindpaw. Rats submitted to sciatic ligation developed cold allodynia at 7 and 14 days post-surgery, whereas the naïve and sham-operated group rats showed no change in cold sensitivity ("Pearson's $\chi^{2}$ test, $\mathrm{P} \leq 0.001$ ). Preop, pre-operation; Postop, post-operation

The remaining miRNAs did not significantly differ in expression among the groups (Fig. 3A; one-way ANOVA, $\mathrm{P}<0.05$ ).

Real-time PCR analysis also indicated that miR-203, miR181a-1* and miR-541* were significantly downregulated among in the bCCI group rats (Fig. 3B; one-way ANOVA, $\mathrm{P}<0.05$, $\mathrm{n}=5$ ), consistent with the microarray analysis. However, none of the above 4 miRNAs showed any significant differences in expression among the naïve, sham-operated and bCCI group rats in the hippocampus or ACC (Fig. 4; one-way ANOVA, $\mathrm{P} \geq 0.05, \mathrm{n}=5$ ).

\section{Discussion}

In this study, we analyzed miRNA expression in different areas of the nociceptive pathway in rats with bCCI compared to naïve and sham-operated rats. We found that the DRG and SDH had a specific and restricted expression of miRNAs that may be associated with the neuropathic pain model. These changes appear to be the result of the specific regulation of miRNAs in individual tissues or organs along the pain transmission pathway, rather than a global change in miRNA or small RNA levels, as the miRNA changes observed after the induction of bCCI were unique to their specific region. Previous studies have determined that miRNA expression in 
Table I. miRNA expression profiling data in the dorsal root ganglion of the rats in the naïve, sham-operated and bCCI groups through one-way ANOVA followed by a Student-Newman-Keuls multiple comparison test.

\begin{tabular}{|c|c|c|c|c|}
\hline \multirow[b]{2}{*}{ Name } & \multicolumn{3}{|c|}{ Average signal in each group } & \multirow{2}{*}{$\begin{array}{c}\text { P-value } \\
\text { one-way ANOVA }\end{array}$} \\
\hline & Naïve & Sham-operated & $\mathrm{bCCI}$ & \\
\hline rno-miR-3559-5p & 0.0022455 & 0.006415 & 0.008763 & 0.024940839 \\
\hline rno-miR-146b & 0.9553484 & 0.8539158 & 1.8513 & 0.046685766 \\
\hline rno-miR-101a & 1.2790858 & 1.9181574 & 2.5265632 & 0.027562292 \\
\hline rno-miR-187 & 0.034373 & 0.0091985 & 0.014633 & 0.008399584 \\
\hline rno-miR-341 & 0.1797986 & 0.170857 & 0.519283 & 0.001411632 \\
\hline rno-miR-33 & 0.6025086 & 0.9127382 & 1.0853938 & 0.03558754 \\
\hline rno-miR-30c & 2.6648084 & 3.891379 & 4.7367974 & 0.010422544 \\
\hline rno-miR-345-5p & 0.1649046 & 0.2183336 & 0.2121974 & 0.024897078 \\
\hline rno-miR-182 & 0.4810526 & 0.5580232 & 1.0227522 & 0.018045938 \\
\hline rno-miR-3583-5p & 0.281121 & 0.0023605 & 0.044797 & 0.03715006 \\
\hline rno-miR-410* & 0.0249235 & 0.005856667 & 0.001947 & 0.008571975 \\
\hline rno-let- $7 i^{*}$ & 0.168998 & 0.2825604 & 0.2546902 & 0.021318724 \\
\hline rno-miR-3597-3p & 0.0094055 & 0.002546 & 0.0018845 & 0.005867282 \\
\hline rno-miR-106b* & 0.0120186 & 0.02215325 & 0.0227986 & 0.035722364 \\
\hline rno-miR-411* & 0.1184006 & 0.1171084 & 0.2318458 & 0.016256798 \\
\hline rno-miR-129-1* & 0.4003384 & 0.2062314 & 0.2269198 & 0.010098178 \\
\hline rno-miR-503 & 0.287767 & 0.2432734 & 0.3782772 & 0.008755002 \\
\hline rno-miR-140 & 0.2425574 & 0.259532 & 0.5147134 & 0.001715675 \\
\hline rno-miR-124 & 5.1789616 & 3.183408 & 6.2611188 & 0.034531422 \\
\hline rno-miR-140* & 0.3500766 & 0.3628198 & 0.6690048 & 0.04393541 \\
\hline rno-miR-195 & 0.6586048 & 0.8834174 & 1.1171146 & 0.02132432 \\
\hline rno-miR-146a* & 0.12624275 & 0.007429667 & 0.02289 & 0.016607437 \\
\hline rno-miR-300-3p & 0.0615604 & 0.070172 & 0.1147072 & 0.025517702 \\
\hline rno-miR-883* & 0.4047586 & 0.2715158 & 0.2310512 & 0.044802193 \\
\hline rno-let-7a & 1.940091 & 1.30881 & 0.5369012 & 0.043364115 \\
\hline rno-miR-100 & 0.7489904 & 0.628444 & 0.3956312 & 0.016141195 \\
\hline rno-miR-20a & 0.3330146 & 0.2114048 & 0.2921848 & 0.00734766 \\
\hline rno-miR-185 & 0.2947272 & 0.1182896 & 0.3695192 & 0.04991285 \\
\hline rno-miR-146a & 0.3531998 & 0.2088992 & 0.482795 & 0.04055271 \\
\hline rno-miR-760-5p & 0.003096 & 0.014082 & 0.012536333 & 0.008632696 \\
\hline rno-miR-28 & 0.112624 & 0.0823878 & 0.0488122 & 0.007563461 \\
\hline rno-miR-154 & 0.0859528 & 0.0647452 & 0.129833 & 0.047474157 \\
\hline rno-miR-337* & 0.06191125 & 0.0365376 & 0.0997064 & 0.005767338 \\
\hline
\end{tabular}

The expression of miR-341 was upregulated in the dorsal root ganglion of rats with bCCI compared to the sham-operated and naïve group rats (fold change $>2, \mathrm{P}<0.05$ ); no significant difference was observed between the naïve and sham-operated group rats. bCCI, bilateral sciatic nerve chronic constriction injury.

the nociceptive system is not only temporally and spatially specific, but also stimulus-dependent $(14,22)$. However, to our knowledge, little is known about the differential expression of miRNAs in the nociceptive pathway during bCCI, and our is the first study to investigate this.

Neuropathic pain is currently under-recognized and undertreated. An increasing body of evidence shows that miRNAs play fundamental roles in neurogenesis, neuron survival, dendritic outgrowth and spine formation (29-31). Aberrant miRNA expression has also been linked to a variety of diseases, including several nervous system diseases (32-34). For example, miR-219 modulates $N$-methyl-D-aspartate (NMDA) receptor-mediated neurobehavioral dysfunction, which is implicated in schizophrenia and autism (35), and the expression of the sensory organ-specific miR-183 family has been shown to be altered following spinal nerve ligation (14). Moreover, 
Table II. miRNA expression profiling data in the SDH of rats in the naïve, sham-operated and bCCI groups through one-way ANOVA followed by a Student-Newman-Keuls multiple comparison test.

\begin{tabular}{|c|c|c|c|c|}
\hline \multirow[b]{2}{*}{ Name } & \multicolumn{3}{|c|}{ Average signal in each group } & \multirow{2}{*}{$\begin{array}{c}\text { P-value } \\
\text { one-way ANOVA }\end{array}$} \\
\hline & Naïve & Sham-operated & bCCI & \\
\hline rno-miR-872 & 0.088160721 & 0.052155876 & 0.027305913 & 0.013864466 \\
\hline rno-miR-196b & 0.121384594 & 0.050332544 & 0.033891158 & 0.004570896 \\
\hline rno-miR-425 & 0.023008365 & 0.01885867 & 0.009661174 & 0.044888683 \\
\hline rno-miR-598-3p & 0.20572949 & 0.119244638 & 0.047441445 & 0.041713364 \\
\hline rno-miR-194 & 0.072227716 & 0.038316478 & 0.021800709 & 0.03436289 \\
\hline rno-miR-203 & 0.024348097 & 0.034445658 & 0.002642154 & 0.025992041 \\
\hline rno-miR-221 & 0.139988347 & 0.070956641 & 0.063008119 & 0.046209376 \\
\hline rno-miR-130b & 0.008131009 & 0.015276097 & 0.00664774 & 0.027954211 \\
\hline rno-miR-181a-1* & 0.027279688 & 0.043180838 & 0.00897442 & 0.009224762 \\
\hline rno-miR-34b & 0.219309204 & 0.098465466 & 0.094511268 & 0.021039538 \\
\hline rno-miR-434* & 0.165626184 & 0.088637773 & 0.064209862 & 0.02409819 \\
\hline rno-miR-485 & 0.134299181 & 0.042754007 & 0.052200311 & 0.039111715 \\
\hline rno-miR-3584-3p & 0.005339837 & 0.028103025 & 0.014496631 & 0.026070911 \\
\hline rno-miR-466c & 0.026500144 & 0.060297098 & 0.010574433 & $4.78 \mathrm{E}-04$ \\
\hline rno-miR-200c & 0.008538573 & 0.019556966 & 0.092025084 & 0.020312184 \\
\hline rno-miR-146a* & 0.04261538 & 0.015903939 & 0.02441009 & 0.022401344 \\
\hline rno-miR-448* & 0.031652286 & 0.049919515 & 0.014945385 & 0.006336924 \\
\hline rno-miR-324-3p & 0.067771106 & 0.033641862 & 0.033705427 & 0.022043822 \\
\hline rno-miR-223 & 0.052215506 & 0.019387663 & 0.028017393 & 0.012133583 \\
\hline rno-miR-218a-2* & 0.011025996 & 0.035931925 & 0.003778643 & 0.04053875 \\
\hline rno-miR-541* & 0.008237646 & 0.014807682 & 0.001920307 & 0.040937595 \\
\hline rno-miR-488 & 0.046445858 & 0.020892955 & 0.02520577 & 0.045676824 \\
\hline rno-miR-10b* & 0.041413909 & 0.021220252 & 0.026583696 & 0.047183 \\
\hline rno-miR-146a & 0.105815309 & 0.060321471 & 0.133724172 & 0.03959339 \\
\hline rno-miR-376c* & 0.089361893 & 0.056806745 & 0.04675663 & 0.04180455 \\
\hline
\end{tabular}

The expression of miR-203, miR-181a-1* and miR-541* was downregulated in the SDH of rats in the bCCI group compared to the shamoperated and naïve group rats (fold change $>2, \mathrm{P}<0.05$ ); no significant difference was observed between the naïve and sham-operated groups bCCI, bilateral sciatic nerve chronic constriction injury; SDH, spinal dorsal horn.

small RNAs have been shown to play critical roles in altering pain thresholds through controlling sodium-channel expression during inflammatory pain (36). Therefore, miRNAs may be novel therapeutic targets for treating these diseases, although we still do not fully understand the molecular mechanisms by which miRNAs regulate gene expression nor do we know the complete repertoire of mRNAs that each miRNA targets.

In this study, using a novel microarray-based approach, we found for the first time that miRNA expression was altered in the DRG and SDH of rats with bCCI. This 6th-generation miRNA array contains more than 1891 capture probes, covering all human, mouse and rat miRNAs annotated in miRBase 16.0 (the older version of the miRNA database), as well as all viral miRNAs related to these species. In addition, this array contains capture probes for 66 new miRPlus ${ }^{\mathrm{TM}}$ human miRNAs. These are proprietary miRNAs not found in
miRBase, and therefore may lead to the discovery of changes in new miRNAs in different disease models.

DRG neurons are primary sensory organs that selectively respond to noxious or potentially tissue-damaging stimuli. They can be sensitized, which is one of the critical mechanisms behind neuropathic pain $(37,38)$. Previous studies have observed that changes in the expression of voltage-gated sodium channel 1.8 and tissue inhibitors of metalloproteinase (TIMPs) occurred in the DRG of rats in a neuropathic pain model $(39,40)$. As shown in our study, miR-341 was upregulated exclusively in the DRG of rats with bCCI, and not in the SDH, hippocampus and ACC. By contrast, miR-541* expression was not detected (even after 40 PCR cycles), indicating that this miRNA either is not expressed, or has a very low expression, in the DRG. Additionally, neither miR-203 nor miR-181a-1* expression was significantly altered in the DRG of the rats with bCCI. Prior to 
A DRG relative expression of different microRNAs (\% control)

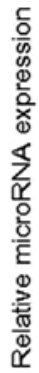

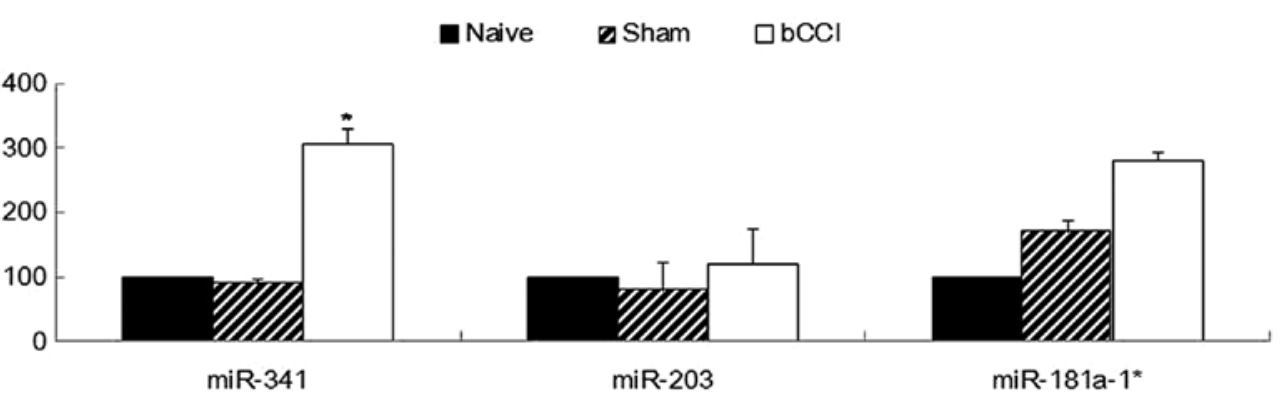

B

SDH relative expression of different microRNAs (\% control)

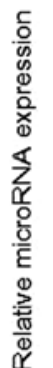

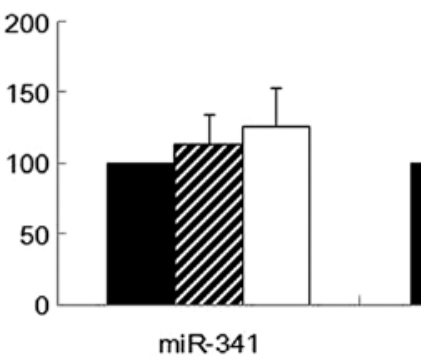

Naive Sham $\square \mathrm{bCCl}$

Figure 3. Relative expression of mature miRNAs in the (A) dorsal root ganglion (DRG) and (B) spinal dorsal horn (SDH) of the naïve, sham-operated and bilateral sciatic nerve chronic constriction injury (bCCI) groups rats determined by real-time-PCR for miR-341, miR-203, miR-181a-1* and miR541*. Data are the means \pm SEM. Data for the sham-operated rats and ligated rats are expressed relative to the naïve rats. Symbols above error bar indicate a significant difference for bCCI group rats (L4-L6 DRG and L2-L4 SDH) compared to the naïve and sham-operated (L4-L6 DRG and L2-L4 SDH) group rats. ("ANOVA, $\mathrm{P}<0.05$ ). The remaining comparisons were not significantly different (ANOVA, $\mathrm{P}>0.05$ ).

$\mathbf{A}$

HIP relative expression of different microRNAs (\% control)

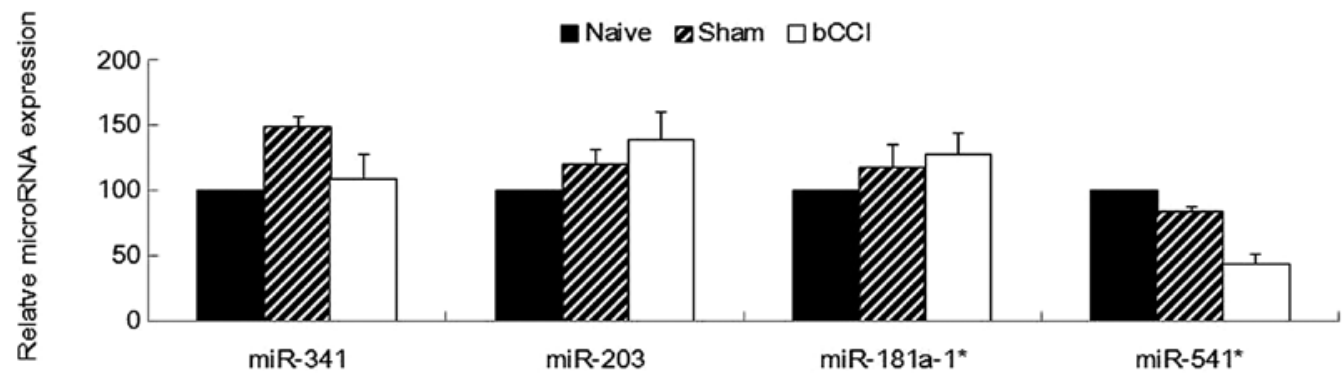

B

ACC relative expression of different microRNAs (\% control)

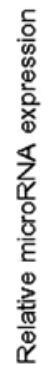

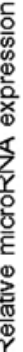

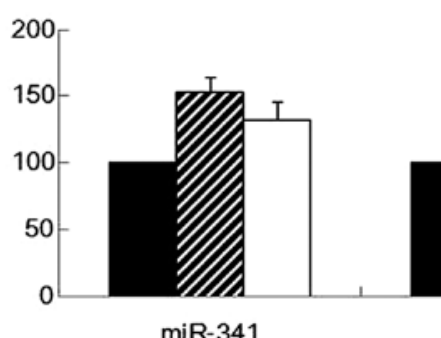

$\operatorname{miR}-341$

Naive Sham $\square \mathrm{bCCl}$

Figure 4. Relative expression of mature miRNAs in the (A) hippocampus (HIP) and (B) anterior cingulate cortex (ACC) of the naïve, sham-operated and bilateral sciatic nerve chronic constriction injury (bCCI) group rats determined by real-time-PCR for miR-341, miR-203, miR-181a-1" and miR541". Data are the means \pm SEM . Data for the sham-operated rats and ligated rats are expressed relative to the naïve rats. The comparisons were not significantly different (ANOVA, $\mathrm{P}>0.05)$. 
our study, Koturbash et al investigated the differential expression of miRNAs associated with X-ray irradiation (41). They found that changes in miRNA expression were tissue-specific in the hippocampus, frontal cortex and cerebellum of female mice. Furthermore, Rao et al observed that tissue-specific RNA interference knocked down Wilms' tumor 1 (WT1) expression in a tissue-specific manner, affecting germ cell survival and spermatogenesis in vivo (42). These results indicate that many miRNAs exhibit tissue- or organ-specific expression patterns and functions in several disease conditions. Our results from profiling the nervous system of rats with bCCI are consistent with this model for miRNA behavior.

miR-203, miR-181a-1* and miR-541* exhibited differential expression only in the SDH of rats with bCCI, but not the DRG, hippocampus or ACC. This suggests that these miRNAs are involved in regulating neuropathic pain, and that their effects are limited to that specific region.

miR-203 has been shown to be downregulated in tumors (43); however, the pathological interactions between neuropathic pain and tumorigenesis require further investigation. Changes in miR-341, miR-541* and miR181a-1* expression have previously only shown a limited association with abnormal states. Therefore, further studies are required to determine the significance of the observed differences in the bCCI model. These findings have implications for neuropathic pain management by miRNA replacement therapy, which can replenish the miRNAs lost or reduced in neuropathic pain by the addition of miRNA mimics. This may also minimize toxicity while retaining potency against the intended miRNA targets.

The 4 miRNAs whose expression was either increased or reduced in the DRG or SDH exhibited no statistically different changes in the hippocampus or ACC. These results suggest that these areas may have their own unique miRNA expression patterns that can influence the development and maintenance of the neuropathic pain condition. However, this conclusion needs to be confirmed by further studies. The realization that the inappropriate production of individual miRNAs in a specific region of the pain transmission pathway contributes to neuropathic pain has reinvigorated antisense oligonucleotide (ASO) drug development $(44,45)$.

In conclusion, in our study, using a rat model of bCCI, we found that miR-341 may play a role in the pathogenesis of neuropathic pain in the DRG, while miR-203, miR-181a- $1^{*}$ and miR-541* may play critical roles in the SDH. Since miRNAs have strong therapeutic potentials and have relatively easy accessibility for systemic or regional delivery in gene therapy, the miRNAs identified in this study may be considered as potential candidates for novel treatment strategies. We expect that our results will help elucidate the molecular mechanisms involved in neuropathic pain and may provide preliminary experimental evidence for the use of miRNAs in the gene therapy treatment of neuropathic pain.

\section{Acknowledgements}

This study was supported by a grant from the National Natural Science Foundation of China (C31070930). We thank Dr Jian Guan of the Pathology Department of the Peking Union Medical College Hospital for her assistance with the animal surgeries.

\section{References}

1. Treede RD, Jensen TS, Campbell JN, et al: Neuropathic pain: redefinition and a grading system for clinical and research purposes. Neurology 70: 1630-1635, 2008.

2. Haanpää M, Attal N, Backonja M, et al: NeuPSIG guidelines on neuropathic pain assessment. Pain 152: 14-27, 2011.

3. Green L and McGhie J: Assessment of acute and chronic pain. Anaesth Intensive Care Med 12: 9-11, 2011.

4. Bie B, Brown DL and Naguib M: Increased synaptic GluR1 subunits in the anterior cingulate cortex of rats with peripheral inflammation. Eur J Pharmacol 653: 26-31, 2011.

5. Boroujerdi A, Zeng J, Sharp K, Kim D, Steward O and Luo ZD: Calcium channel alpha-2-delta-1 protein upregulation in dorsal spinal cord mediates spinal cord injury-induced neuropathic pain states. Pain 152: 649-655, 2011

6. Chou CW, Wong GT, Lim G, et al: Peripheral nerve injury alters the expression of NF-kappaB in the rat's hippocampus. Brain Res 1378: 66-71, 2011.

7. Emery EC, Young GT, Berrocoso EM, Chen L and McNaughton PA: HCN2 ion channels play a central role in inflammatory and neuropathic pain. Science 333: 1462-1466, 2011.

8. Jaggi AS and Singh N: Role of different brain areas in peripheral nerve injury-induced neuropathic pain. Brain Res 1381: 187-201, 2011.

9. Vogt BA: Pain and emotion interactions in subregions of the cingulate gyrus. Nat Rev Neurosci 6: 533-544, 2005.

10. Hodgdon KE, Hingtgen CM and Nicol GD: Dorsal root ganglia isolated from $\mathrm{Nf}^{+/-}$mice exhibit increased levels of mRNA expression of voltage-dependent sodium channels. Neuroscience 206: 237-244, 2012.

11. Kim DS, Figueroa KW, Li KW, Boroujerdi A, Yolo T and Luo ZD: Profiling of dynamically changed gene expression in dorsal root ganglia post peripheral nerve injury and a critical role of injury-induced glial fibrillary acetic protein in maintenance of pain behaviors. Pain 143: 114-122, 2009.

12. Cady RJ, Glenn JR, Smith KM and Durham PL: Calcitonin gene-related peptide promotes cellular changes in trigeminal neurons and glia implicated in peripheral and central sensitization. Mol Pain 7: 94, 2011.

13. Uchida H, Ma L and Ueda H: Epigenetic gene silencing underlies C-fiber dysfunctions in neuropathic pain. J Neurosci 30: 4806-4814, 2010.

14. Aldrich BT, Frakes EP, Kasuya J, Hammond DL and Kitamoto T: Changes in expression of sensory organ-specific microRNAs in rat dorsal root ganglia in association with mechanical hypersensitivity induced by spinal nerve ligation. Neuroscience 164: 711-723, 2009.

15. Bak M, Silahtaroglu A, Moller M, et al: MicroRNA expression in the adult mouse central nervous system. RNA 14: 432-444, 2008

16. Bastian I, Tam Tam S, Zhou X-F, et al: Differential expression of microRNA-1 in dorsal root ganglion neurons. Histochem Cell Biol 135: 37-45, 2010.

17. von Schack D, Agostino MJ, Murray BS, et al: Dynamic changes in the microRNA expression profile reveal multiple regulatory mechanisms in the spinal nerve ligation model of neuropathic pain. PLoS One 6: e17670, 2011.

18. Chiang HR, Schoenfeld LW, Ruby JG, et al: Mammalian microR NAs: experimental evaluation of novel and previously annotated genes. Genes Dev 24: 992-1009, 2010.

19. Hua YJ, Tang ZY, Tu K, et al: Identification and target prediction of miRNAs specifically expressed in rat neural tissue. BMC Genomics 10: 214, 2009.

20. Kosik KS: The neuronal microRNA system. Nat Rev Neurosci 7: 911-920, 2006.

21. Wheeler G, Ntounia-Fousara S, Granda B, Rathjen T and Dalmay T: Identification of new central nervous system specific mouse microRNAs. FEBS Lett 580: 2195-2200, 2006.

22. Kusuda R, Cadetti F, Ravanelli MI, et al: Differential expression of microRNAs in mouse pain models. Mol Pain 7: 17, 2011.

23. Harraz MM, Dawson TM and Dawson VL: MicroRNAs in Parkinson's disease. J Chem Neuroanat 42: 127-130, 2011.

24. Zhang HY, Zheng SJ, Zhao JH, et al: MicroRNAs 144, 145 , and 214 are down-regulated in primary neurons responding to sciatic nerve transection. Brain Res 1383: 62-70, 2011.

25. Datta S, Chatterjee K, Kline RH and Wiley RG: Behavioral and anatomical characterization of the bilateral sciatic nerve chronic constriction (bCCI) injury: correlation of anatomic changes and responses to cold stimuli. Mol Pain 6: 7, 2010. 
26. Vierck CJ, Acosta-Rua AJ and Johnson RD: Bilateral chronic constriction of the sciatic nerve: a model of long-term cold hyperalgesia. J Pain 6: 507-517, 2005.

27. Chaplan SR, Bach FW, Pogrel JW, Chung JM and Yaksh TL: Quantitative assessment of tactile allodynia in the rat paw. J Neurosci Methods 53: 55-63, 1994.

28. Schmittgen TD and Livak KJ: Analyzing real-time PCR data by the comparative CT method. Nat Protoc 3: 1101-1108, 2008.

29. Krichevsky AM, King KS, Donahue CP, Khrapko K and Kosik KS: A microRNA array reveals extensive regulation of microRNAs during brain development. RNA 9: 1274-1281,2003.

30. Ambros V: The functions of animal microRNAs. Nature 431: 350-355, 2004.

31. Schratt GM, Tuebing F, Nigh EA, et al: A brain-specific microRNA regulates dendritic spine development. Nature 439 283-289, 2006.

32. Mercader JM, Gonzalez JR, Lozano JJ, et al: Aberrant brain microRNA target and miRISC gene expression in the anx/anx anorexia mouse model. Gene 497: 181-190, 2012.

33. Wei L, Wang M, Qu X, et al: Differential expression of microRNAs during allograft rejection. Am J Transplant 12: 1113-1123, 2012.

34. Liu PT, Wheelwright M, Teles R, et al: MicroRNA-21 targets the vitamin D-dependent antimicrobial pathway in leprosy. Nat Med 18: 267-273, 2012.

35. Kocerha J,Faghihi MA,Lopez-Toledano MA, etal:MicroRNA-219 modulates NMDA receptor-mediated neurobehavioral dysfunction. Proc Natl Acad Sci USA 106: 3507-3512, 2009.

36. Zhao J, Lee MC, Momin A, et al: Small RNAs control sodium channel expression, nociceptor excitability, and pain thresholds. J Neurosci 30: 10860-10871, 2010.
37. Kuner R: Central mechanisms of pathological pain. Nature Med 16: 1258-1266, 2010.

38. Gold MS and Gebhart GF: Nociceptor sensitization in pain pathogenesis. Nature Med 16: 1248-1257, 2010.

39. Ruangsri S, Lin A, Mulpuri Y, Lee K, Spigelman I and Nishimura I: Relationship of axonal voltage-gated sodium channel 1.8 (NaV1.8) mRNA accumulation to sciatic nerve injury-induced painful neuropathy in rats. J Biol Chem 286: 39836-39847, 2011.

40. Huang B, Zhao X, Zheng LB, Zhang L, Ni B and Wang YW: Different expression of tissue inhibitor of metalloproteinase family members in rat dorsal root ganglia and their changes after peripheral nerve injury. Neuroscience 193: 421-428, 2011.

41. Koturbash I, Zemp F, Kolb B and Kovalchuk O: Sex-specific radiation-induced microRNAome responses in the hippocampus, cerebellum and frontal cortex in a mouse model. Mutat Res 722: 114-118, 2011.

42. Rao MK, Pham J, Imam JS, et al: Tissue-specific RNAi reveals that WT1 expression in nurse cells controls germ cell survival and spermatogenesis. Genes Dev 20: 147-152, 2006.

43. Bo J, Yang G, Huo K, et al: microRNA-203 suppresses bladder cancer development by repressing bcl-w expression. FEBS J 278: 786-792, 2011.

44. Elmén J, Lindow M, Schütz S, et al: LNA-mediated microRNA silencing in non-human primates. Nature 452: 896-899, 2008.

45. Broderick JA and Zamore PD: MicroRNA therapeutics. Gene Ther 18: 1104-1110, 2011. 